Proceedings

\title{
Boltzmann Entropy, the Holographic Bound and Newtonian Cosmology ${ }^{+}$
}

\author{
Pedro Fernández De Córdoba * and Jose Maria Isidro San Juan \\ Instituto Universitario de Matemática Pura y Aplicada, Universidad Politécnica de Valencia, 46022 Valencia, \\ Spain; joissan@mat.upv.es \\ * Correspondence: pfernandez@mat.upv.es \\ † Presented at the 4th International Electronic Conference on Entropy and Its Applications, \\ 21 November-1 December 2017; Available online: http:/ / sciforum.net/conference/ecea-4.
}

Published: 21 November 2017

\begin{abstract}
The holographic principle sets an upper bound on the total (Boltzmann) entropy content of the Universe at around $10^{123} k_{B}$ ( $k_{B}$ being Boltzmann's constant). In this work we point out the existence of a remarkable duality between nonrelativistic quantum mechanics on the one hand, and Newtonian cosmology on the other. Specifically, nonrelativistic quantum mechanics has a quantum probability fluid that exactly mimics the behaviour of the cosmological fluid, the latter considered in the Newtonian approximation. One proves that the equations governing the cosmological fluid (the Euler equation and the continuity equation) become the very equations that govern the quantum probability fluid after applying the Madelung transformation to the Schroedinger wavefunction. Under the assumption that gravitational equipotential surfaces can be identified with isoentropic surfaces, this model allows for a simple computation of the gravitational entropy of a Newtonian Universe.
\end{abstract}

Keywords: gravitational entropy; holographic principle; emergent spacetime

\section{Introduction}

There is a widespread certainty that the continuum description of spacetime as provided by general relativity must necessarily break down at very short length scales and/or very high curvatures. A number of very different approaches to an eventual theory of quantum gravity have been presented in the literature; these candidate theories are too varied and too extensive to summarise here. On the whole, the picture that emerges is that of a continuum description after some appropriate coarse graining of some underlying degrees of freedom. Even if the precise nature of the latter is unknown yet, one can still make progress following a thermodynamical approach: one ignores large amounts of detailed knowledge (say, the precise motions followed by the atoms of a gas) while concentrating only on a few coarse-grained averages (say, the overall pressure exerted by the atoms of a gas on the container walls). This way of approaching the problem has come to be called the emergent approach.

In the emergent approach to spacetime, gravity qualifies as an entropic force. This means that we do not know the fundamental degrees of freedom underlying gravity, but their overall macroscopic effect is that of driving the system under consideration in the direction of increasing entropy. If gravitational forces are entropy gradients, then gravitational equipotential surfaces can be identified with isoentropic surfaces. We will consider a density of particles representing the (baryonic and dark) matter contents of a hypothetical Newtonian Universe. This volume density will be identified with the squared modulus of a nonrelativistic wavefunction $\psi$ satisfying the Schroedinger equation. Let $U$ denote the gravitational potential. Once dimensions are corrected (using $\hbar$ and $k_{B}$ ), the expectation value $\langle\psi|U| \psi\rangle$ becomes a measure of the gravitational entropy of the Universe when the matter is described by the wavefunction $\psi$. 


\section{Methods}

In Newtonian cosmology, the Universe is regarded as being subject to a gravitational potential $U$ satisfying the Poisson equation

$$
\nabla^{2} U=4 \pi G \rho .
$$

The matter content (baryonic and dark matter) is modelled as an ideal fluid satisfying the continuity equation and the Euler equation,

$$
\frac{\partial \rho}{\partial t}+\nabla \cdot(\rho \mathbf{v})=0, \quad \frac{\partial \mathbf{v}}{\partial t}+(\mathbf{v} \cdot \nabla) \mathbf{v}+\frac{1}{\rho} \nabla p-\mathbf{F}=0 .
$$

The cosmological principle requires that the velocity field $\mathbf{v}$ be everywhere proportional to the position vector $\mathbf{r}$. This requirement is equivalent to Hubble's law [1-3], which can be described phenomenologically by the harmonic potential

$$
U_{\text {Hubble }}(\mathbf{r})=-\frac{H_{0}^{2}}{2} \mathbf{r}^{2}
$$

Hubble's constant $H_{0}$ is an angular frequency; the negative sign implies that this potential is repulsive. Accordingly, $U_{\text {Hubble }}$ satisfies the Poisson Equation (1) with a negative mass density.

Schroedinger quantum mechanics can also be understood in terms of an ideal fluid, the quantum probability fluid. Following Madelung one factorises the nonrelativistic wavefunction $\psi$ into amplitude and phase:

$$
\psi=\exp \left(\frac{\mathcal{S}}{2 k_{B}}+\mathrm{i} \frac{\mathcal{I}}{\hbar}\right) .
$$

The amplitude $\exp \left(\mathcal{S} / 2 k_{B}\right)$ is a real exponential; one can invoke Boltzmann's principle to regard $\mathcal{S}$ as a Boltzmann entropy of the matter described by $\psi-$ not to be confused with the gravitational entropy $\mathcal{S}_{g}$ in Equation (16) below. It will also be convenient to define a dimensionless Boltzmann entropy $S:=\mathcal{S} / 2 k_{B}$. The phase $\exp (\mathrm{i} \mathcal{I} / \hbar)$ is the complex exponential of the classical-mechanical action integral $\mathcal{I}$. Substituting the Ansatz Equation (4) into the Schroedinger equation for $\psi$, one arrives at a set of two equations. One of them is the continuity equation for the quantum probability fluid,

$$
\frac{\partial S}{\partial t}+\frac{1}{m} \nabla S \cdot \nabla \mathcal{I}+\frac{1}{2 m} \nabla^{2} \mathcal{I}=0,
$$

where

$$
\mathbf{v}:=\frac{1}{m} \nabla \mathcal{I}, \quad \rho=\mathrm{e}^{2 S} .
$$

The second equation obtained is known as the quantum Hamilton-Jacobi equation:

$$
\frac{\partial \mathcal{I}}{\partial t}+\frac{1}{2 m}(\nabla \mathcal{I})^{2}+V+\mathcal{Q}=0,
$$

where $V$ is the external potential present in the Schroedinger equation (we recall that the dimensions of $U$ in Equations (1) and (3) are velocity squared, whereas those of $V$ in Equation (7) are mass times velocity squared). Above,

$$
\mathcal{Q}:=-\frac{\hbar^{2}}{2 m}\left[(\nabla S)^{2}+\nabla^{2} S\right]
$$

is known as the quantum potential.

Finally we need to derive an Euler equation for the quantum probability. This is achieved by taking the gradient of Equation (7):

$$
\frac{\partial \mathbf{v}}{\partial t}+(\mathbf{v} \cdot \nabla) \mathbf{v}+\frac{1}{m} \nabla \mathcal{Q}+\frac{1}{m} \nabla V=0 .
$$


Comparison between Equations (2) and (9) produces a bijective correspondence between the quantum probability fluid and the cosmological fluid. Which suggests that, given the cosmological fluid in the Newtonian approximation, we use nonrelativistic quantum mechanics as an equivalent description thereof. In this description by means of a quantum wavefunction $\psi$, the amount of mass $m_{V}$ contained within a volume $V$ equals $m_{V}=m \int_{V} \mathrm{~d}^{3} x|\psi|^{2}$; the whole observable Universe is regarded as a sphere of radius $R_{0}$. Considering the Universe as a sphere with finite radius has the added bonus that the instabilities [4] due to the negative sign of the potential Equation (3) are avoided naturally.

In view of Hubble's law Equation (3) it is reasonable to consider the effective Hamiltonian

$$
H_{\text {eff }}=-\frac{\hbar^{2}}{2 m} \nabla^{2}-\frac{k_{\text {eff }}}{2} \mathbf{r}^{2}, \quad k_{\text {eff }}=m H_{0}^{2}
$$

as governing the overall expansion of the Universe, at least within the Newtonian limit. As a first approximation it will also be useful to consider the free Hamiltonian

$$
H_{\text {free }}=-\frac{\hbar^{2}}{2 m} \nabla^{2}
$$

Their respective eigenfunctions are readily obtained in spherical coordinates. For Equation (11) we have the free spherical waves

$$
\psi_{\kappa 00}(r, \theta, \varphi)=\frac{1}{\sqrt{4 \pi R_{0}}} \frac{1}{r} \exp (\mathrm{i} \kappa r), \quad \kappa \in \mathbb{R},
$$

normalised within a sphere of radius $R_{0}$, and carrying zero angular momentum as required by the cosmological principle. For the Hubble Hamiltonian Equation (10) one finds the exact eigenfunctions [5]

$$
\psi_{\alpha}^{(1)}(r, \theta, \varphi)=\frac{N_{\alpha}^{(1)}}{\sqrt{4 \pi}} \exp \left(\frac{\mathrm{i} \beta^{2} r^{2}}{2}\right){ }_{1} F_{1}\left(\frac{3}{4}-\frac{\mathrm{i} \alpha}{4}, \frac{3}{2} ;-\mathrm{i} \beta^{2} r^{2}\right)
$$

and

$$
\psi_{\alpha}^{(2)}(r, \theta, \varphi)=\frac{N_{\alpha}^{(2)}}{\sqrt{4 \pi}} \frac{1}{r} \exp \left(\frac{\mathrm{i} \beta^{2} r^{2}}{2}\right){ }_{1} F_{1}\left(\frac{1}{4}-\frac{\mathrm{i} \alpha}{4}, \frac{1}{2} ;-\mathrm{i} \beta^{2} r^{2}\right) .
$$

They also carry vanishing angular momentum, $N_{\alpha}^{(1)}$ and $N_{\alpha}^{(2)}$ being radial normalisation factors. Above, ${ }_{1} F_{1}$ is the confluent hypergeometric function, and the parameters $\alpha, \beta$ are given by

$$
\alpha:=\frac{2 E}{\hbar H_{0}}, \quad \beta^{4}:=\frac{m^{2} H_{0}^{2}}{\hbar^{2}}
$$

with $E$ the energy eigenvalue in $H_{\text {eff }} \psi=E \psi$.

\section{Results and Discussion}

Our previous reasoning leads naturally to the operator $\mathbf{R}^{2}=X^{2}+Y^{2}+Z^{2}$ as a measure of the amount of gravitational entropy contained within a Newtonian Universe in which the Hubble repulsion arises as the net force. Specifically, the operator

$$
\mathcal{S}_{g}:=\mathcal{N} \frac{k_{B} m H_{0}}{\hbar} \mathbf{R}^{2}
$$

is dimensionally an entropy; a dimensionless factor $\mathcal{N}$ is of course left undetermined.We call $\mathcal{S}_{g}$ the gravitational entropy operator.

We can now compute the expectation value of the entropy $\mathcal{S}_{g}$ in the free eigenstates Equation (12) and in the Hubble eigenstates Equations (13) and (14). For the free waves Equation (12) one finds 


$$
\left\langle\psi_{\kappa 00}\left|\mathbf{R}^{2}\right| \psi_{\kappa 00}\right\rangle=\frac{R_{0}^{2}}{3} .
$$

Substituting the known values [6] of the cosmological data $m, H_{0}, R_{0}$ into Equations (16) and (17) we arrive at the estimate [7]

$$
\left\langle\psi_{\kappa 00}\left|\mathcal{S}_{g}\right| \psi_{\kappa 00}\right\rangle=10^{123} k_{B}
$$

Above we have set $\mathcal{N}=3 / 2.6$. Our result Equation (18) saturates the upper bound set by the holographic principle. A finer estimate is obtained using the Hubble waves Equations (13) and (14). After some numerical approximations one finds

$$
\left\langle\psi_{\alpha}^{(1)}\left|\mathbf{R}^{2}\right| \psi_{\alpha}^{(1)}\right\rangle=\frac{R_{0}^{2}}{2 \ln \left(\beta R_{0}\right)}=\left\langle\psi_{\alpha}^{(2)}\left|\mathbf{R}^{2}\right| \psi_{\alpha}^{(2)}\right\rangle .
$$

This leads to [5]

$$
\left\langle\psi_{\alpha}^{(1)}\left|\mathcal{S}_{g}\right| \psi_{\alpha}^{(1)}\right\rangle=10^{120} k_{B}=\left\langle\psi_{\alpha}^{(2)}\left|\mathcal{S}_{g}\right| \psi_{\alpha}^{(2)}\right\rangle
$$

upon taking $\mathcal{N}=1 / 6$. This new theoretical estimate lies three orders of magnitude below the holographic bound, thus representing a considerable improvement on the estimate obtained from the free waves.

\section{Conclusions}

The holographic principle sets an upper bound of approximately $10^{123} k_{B}$ on the entropy content of the Universe. Some phenomenological estimates [8] place the actual value at around $10^{104} k_{B}$, gravitational entropy (and, in particular, black holes) representing the largest single contributors to the entropy budget of the Universe. Although Newtonian cosmology does allow for black holes, the many simplifications made by our elementary model necessarily leave out some essential physics of the Universe. Nevertheless, our toy model succeeds in capturing some key elements of reality. For example, the upper bound set by the holographic principle is always respected, even by such a crude approximation as the free waves Equation (12). The Hubble waves Equations (13) and (14) represent a considerable improvement on the free waves, as they reduce the expectation value of the entropy by three orders of magnitude.

Acknowledgments: This research was supported by grant no. ENE2015-71333-R (Spain).

Conflicts of Interest: The authors declare no conflict of interest.

\section{References}

1. Hubble, E. A Relation between Distance and Radial Velocity among Extra-Galactic Nebulae. Proc. Nat. Acad. Sci. USA 1929, 15, 168-173.

2. Perlmutter, S.; Aldering, G.; Deustua, S.; Fabbro, S.; Goldhaber, G.; Groom, D.E.; Kim, A.G.; Kim, M.Y.; Knop, R.A.; Nugent, P.; et al. Cosmology from Type Ia Supernovae. Bull. Am. Astron. Soc. 1997, $29,1351$.

3. Riess, A.G.; Filippenko, A.V.; Challis, P.; Clocchiatti, A.; Diercks, A.; Garnavich, P.M.; Gilliland, R.L.; Hogan, C.J.; Jha, S.; Kirshner, R.P.; et al. Observational Evidence from Supernovae for an Accelerating Universe and a Cosmological Constant. Astron. J. 1998, 116, 1009-1038.

4. Broadbridge, P.; Zulkowski, P. Dark Energy States from Quantization of Boson Fields in a Universe with Unstable Modes. Rep. Math. Phys. 2006, 57, 27-40.

5. Fernández de Córdoba, P.; Isidro, J.M. On the Holographic Bound in Newtonian Cosmology. arXiv 2017, arXiv:1710.00507.

6. Ade, P.A.; Aghanim, N.; Arnaud, M.; Ashdown, M.; Aumont, J.; Baccigalupi, C.; Banday, A.J.; Barreiro, R.B.; Bartlett, J.G.; Bartolo, N.; et al. Planck 2015 Results. XIII. Cosmological Parameters. Astron. Astrophys. 2016, 594, A13. 
7. Cabrera, D.; Fernández de Córdoba, P.; Isidro, J.M. Boltzmann Entropy of a Newtonian Universe. Entropy 2017, 19, 212, arXiv:1703.08082.

8. Egan, C.; Lineweaver, C. A Larger Estimate of the Entropy of the Universe. Astrophys. J. 2010, 710, 1825-1834.

(C) 2018 by the authors. Licensee MDPI, Basel, Switzerland. This article is an open access article distributed under the terms and conditions of the Creative Commons Attribution (CC BY) license (http://creativecommons.org/licenses/by/4.0/). 